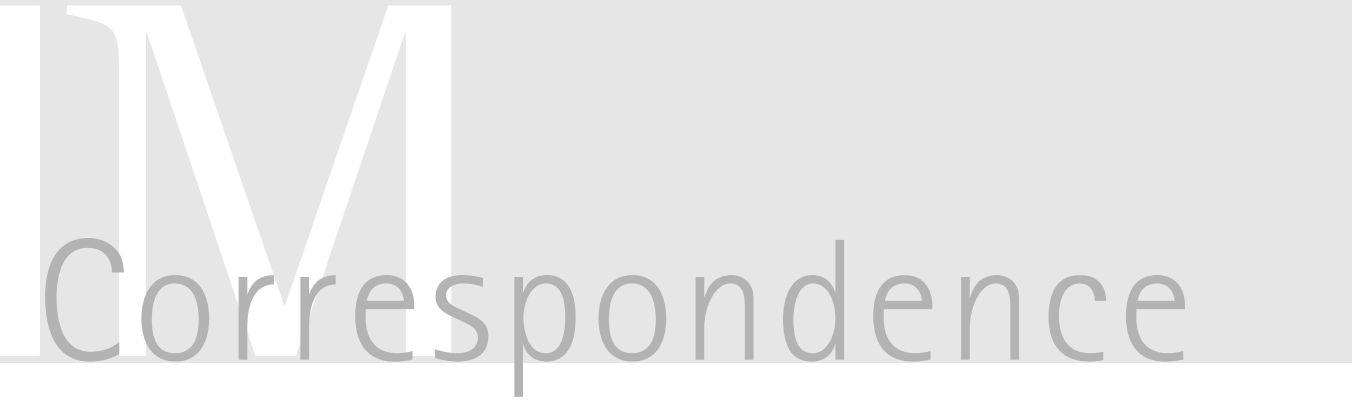

\title{
Emergence of vancomycin-intermediate Staphylococcus species in southern India
}

Since the emergence of meticillin-resistant Staphylococcus aureus (MRSA), vancomycin has been the only uniformly effective treatment for staphylococcal infections in India, teicoplanin and linezolid not being commonly used. The incidence of vancomycin-intermediate $S$. aureus (VISA) and vancomycin-resistant $S$. aureus (VRSA) has been increasing in various parts of the world. The first clinical isolate of VRSA was reported from the United States in 2002 (CDC, 2002). More recently, some workers have reported vancomycin-resistant staphylococcal strains from Brazil (Palazzo et al., 2005), Jordan (Bataineh, 2006) and India (Tiwari \& Sen, 2006). Vancomycin intermediateresistant Staphylococcus haemolyticus has been reported previously (Schwalbe et al., 1987), and perhaps this is a critical antecedent of VISA. As documented reports of VISA/VRSA in India are very few (Assadullah et al., 2003), we decided to monitor the vancomycin susceptibility of clinical meticillin-resistant Staphylococcus spp. in the tertiary care teaching hospital, JIPMER.

A total of 261 staphylococcal isolates consisting of 141 S. aureus isolates and 120 coagulase-negative staphylococci (CNS) were included. The clinical specimens included wound swabs, catheters, pus, urine, sputum and blood from inpatients and outpatients of the tertiary care teaching hospital, JIPMER. Staphylococci were identified according to standard methodology (Koneman et al., 2006). The identity of the isolates was confirmed by a latex agglutination test, the HiStaph Latex test, performed according to the protocols supplied by the manufacturer (HiMedia). Staphylococci were obtained either as pure culture or as part of a polymicrobial infection. All S. aureus isolates and 30 CNS were subjected to susceptibility testing by the Kirby-Bauer disc diffusion method, the Brain Heart Infusion vancomycin screen agar test $\left(6 \mu \mathrm{g}\right.$ vancomycin $\left.\mathrm{ml}^{-1}\right)$ and MIC testing of vancomycin by the agar dilution method. S. aureus isolates were screened for oxacillin resistance by the oxacillin screen agar test $(6 \mu \mathrm{g}$ oxacillin $\mathrm{ml}^{-1}$ in Mueller-Hinton agar) as well as the oxacillin disc diffusion test. The tests were performed according to NCCLS guidelines (NCCLS, 2000). MICs of the antimicrobials for the isolates were crosschecked by the HiComb MIC test (HiMedia) performed according to the protocols supplied by the manufacturer. Quality control was performed using the $S$. aureus ATCC 29213 strain. The MIC is the value at which the zone converges on the comb-like projections of the strips. Isolates with a vancomycin MIC between 4 and $8 \mu \mathrm{g} \mathrm{ml}^{-1}$ were identified as vancomycinintermediate (CLSI, 2006). The oxacillin screen agar test and oxacillin disc diffusion test of 141 S. aureus isolates revealed 102 to be resistant to oxacillin. The oxacillin disc diffusion test on CNS picked up 21 resistant isolates. Of 102 oxacillin-resistant $S$. aureus isolates, one was found to be a VISA strain (MIC $5 \mu \mathrm{g} \mathrm{ml}^{-1}$ ) (Fig. 1). Of
21 oxacillin-resistant CNS, five isolates were vancomycin-intermediate (four isolates with MIC of $5 \mu \mathrm{g} \mathrm{ml}^{-1}$ and one with MIC of $10 \mu \mathrm{g} \mathrm{ml}^{-1}$ ) (Fig. 2). The clinical details of the patients from whom vancomycin-intermediate staphylococci were isolated are given in Table 1. The age of the patients infected with vancomycinintermediate staphylococci ranged from 15 to 54 years; four were females and two were males. All of these 38 isolates were isolated from hospitalized patients and were also found to be resistant to several other antimicrobials such as gentamicin, ciprofloxacin and erythromycin. The MICs of vancomycin for the rest of the staphylococci ranged from 0.5 to 2 $\mu \mathrm{g} \mathrm{ml}^{-1}$. This study reveals for the first time the emergence of vancomycinintermediate staphylococcal isolates in southern India. To understand this emergence of VISA, one has to look at the increased incidence of MRSA in this hospital. In a preliminary report in 1997, among 120 consecutively collected isolates

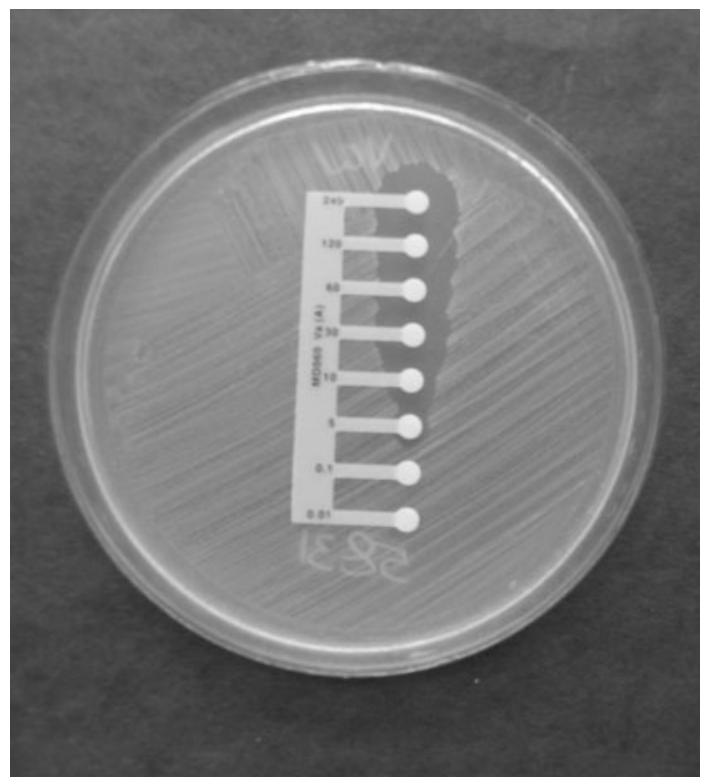

Fig. 1. HiComb MIC test for $S$. aureus, showing a vancomycin MIC of $5 \mu \mathrm{g} \mathrm{ml}^{-1}$. 


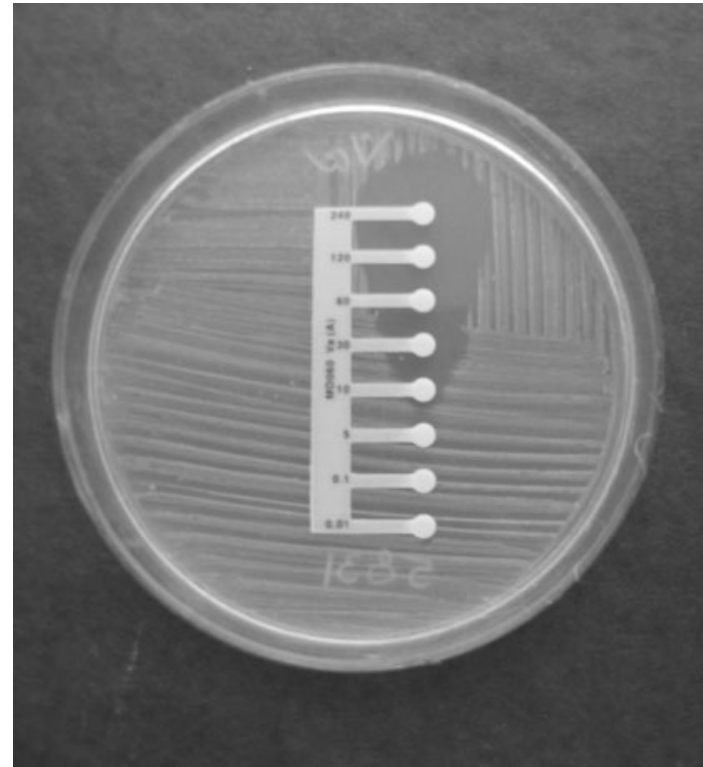

Fig. 2. HiComb MIC test for $S$. haemolyticus, showing a vancomycin MIC of $10 \mu \mathrm{g} \mathrm{ml}^{-1}$.

Table 1. Details of vancomycin-intermediate staphylococcal isolates

\begin{tabular}{|lccll|}
\hline Isolate & $\begin{array}{c}\text { No. of } \\
\text { isolates }\end{array}$ & $\begin{array}{c}\text { MIC of vancomycin } \\
\left(\boldsymbol{\mu} \mathbf{~ m l}^{-\mathbf{1}}\right)\end{array}$ & Specimen & Ward/OPD \\
\hline S. aureus & 1 & 5 & Pus & Surgery ward \\
S. haemolyticus & 1 & 5 & Tracheal aspirate & Intensive care unit \\
S. haemolyticus & 1 & 10 & Catheter tip & Intensive care unit \\
S. lugdunensis & 3 & 5 & Pus & Post-operative ward \\
\hline
\end{tabular}

of S. aureus, only 24 were MRSA (Shankar et al., 1997). During the period 2004-2006, among 2468 isolates of $S$. aureus, 878 isolates were MRSA. All isolates of MRSA were found to be susceptible to vancomycin with MICs of $<2 \mu \mathrm{g} \mathrm{ml}^{-1}$. The continued increase in the incidence of MRSA has led to widespread use of vancomycin in the treatment of staphylococcal infections. This might have induced a selection pressure for the emergence. Like the earlier findings (Schwalbe et al., 1987), S. haemolyticus appears to be the critical antecedent of VISA. The emergence of vancomycinintermediate staphylococcal isolates is of great concern. The large scale of development and subsequent spread of resistance to vancomycin is perceived as a fearsome threat to the already challenging therapy of MRSA. This is a matter of concern because there are hardly any treatment options available in this country. This study emphasizes the need for continuous monitoring of MIC levels of vancomycin in MRSA, and the importance of its prudent use and the infectioncontrol practices to prevent its transmission.

\section{G. A. Menezes, ${ }^{1}$ B. N. Harish, ${ }^{1}$ S. Sujatha, ${ }^{1}$ K. Vinothini ${ }^{2}$ and S. C. Parija ${ }^{1}$}

${ }^{1}$ Department of Microbiology, Jawaharlal Institute of Postgraduate Medical
Education and Research (JIPMER), Pondicherry-605006, India

${ }^{2}$ Department of Botany and Microbiology, A.V.V.M. Sri Pushpam College

(Autonomous) Poondi - 613503,

Thanjavur Dist., India

Correspondence: B. N. Harish (drbnharish@yahoo.com)

Assadullah, S., Kakru, D. K., Thoker, M. A., Bhat, P. A., Hussain, N. \& Shah, A. (2003).

Emergence of low level vancomycin resistance in MRSA. Indian J Med Microbiol 21, 196-198.

Bataineh, H. A. (2006). Resistance of Staphylococcus aureus to vancomycin in Zarqa, Jordan. Pak J Med Sci 22, 144-148.

Centers for Disease Control and Prevention (CDC) (2002). Staphylococcus aureus resistant to vancomycin - United States. MMWR Morb Mortal Wkly Rep 51, 565-567.

Clinical and Laboratory Standards Institute (2006). Performance Standards for Antimicrobial Susceptibility Testing, 16th informational supplement. M100-S16. Wayne, PA: Clinical and Laboratory Standards Institute.

Koneman, E. W., Allen, S. D., Janda, W. M. \& Schreckenberger, P. C. (2006). The Gram positive cocci. I. Staphylococci and related organisms. In Color Atlas and Textbook of Diagnostic Microbiology, 6th edn, pp. 624-662. Edited by E. W. Koneman, S. D. Allen, W. M. Janda, P. C. Schreckenberger, G. W. Propcop, G. L. Woods \& W. C. Winn, Jr. Philadelphia: Lippincott-Raven.

NCCLS (2000). Methods for Dilution Antimicrobial Susceptibility Tests for Bacteria that Grow Aerobically. Approved Standard M7-A5. Wayne, PA: National Committee for Clinical Laboratory Standards.

Palazzo, I. C. V., Araujo, M. L. C. \& Darini, A. L. C. (2005). First report of vancomycin-resistant staphylococci isolated from healthy carriers in Brazil. J Clin Microbiol 43, 179-185.

Schwalbe, R. S., Stapleton, J. T. \& Gilligan, P. H. (1987). Emergence of vancomycin resistance in coagulase negative staphylococci. $N$ Engl J Med 316, 927-931.

Shankar, C. U., Harish, B. N., Kumar, P. M. U. \& Navaneeth, B. V. (1997). Prevalence of methicillin resistant Staphylococcus aureus in JIPMER hospital - a preliminary report. Indian J Med Microbiol 15, 137-138.

Tiwari, H. K. \& Sen, M. R. (2006). Emergence of vancomycin resistant Staphylococcus aureus (VRSA) from a tertiary care hospital from northern part of India. BMC Infect Dis 6, 156. 